\title{
m-commuting Additive Maps on Upper Triangular Matrices Rings
}

\section{Driss Aiat Hadj Ahmed}

Centre Régional des Métiers d'Education et de Formation (CRMEF), Tangier, Morocco e-mail: ait_hadj@yahoo.com

\begin{abstract}
Let $T_{n}(R)$ be the upper triangular matrix ring over a unital commutative ring whose characteristic is not a divisor of $m$. Suppose that $f: T_{n}(R) \rightarrow T_{n}(R)$ is an additive map such that $X^{m} f(X)=f(X) X^{m}$ for all $X \in T_{n}(R)$, where $m \geq 1$ is an integer. We consider the problem of describing the form of the map $X \rightarrow f(X)$.
\end{abstract}

\section{Introduction and Results}

For a ring $R$ we say that the map $f: R \rightarrow R$ is commuting if $[f(x), x]=0$ for every $x \in R$, where $[a, b]=a b-b a$ denotes the standard commutator. The study of such maps was inspired by Posner [13] who proved that if a prime ring has a nonzero centralizing derivation, then it must be commutative. This theorem was generalized in many ways (see for instance $[1,10,11,12,15]$ ). The first general result regarding commuting maps comes from Brešar [4] who has shown that additive commuting maps $f$ over a simple unital ring $R$ must be of the form $f(x)=\lambda x+\mu: R \rightarrow Z(R)$, where $Z(R)$ denotes the center of $R$. This form is usually called a standard form for the commuting map.

There are plenty of results on commuting maps (for example $[7,8,9,16]$ ) and the

Received: July 28, 2019; Accepted: September 5, 2019

2010 Mathematics Subject Classification: 47B47, 15A27, 15A04.

Keywords and phrases: commuting maps, upper triangular matrices rings, additive maps.

Copyright (C) 2019 Driss Aiat Hadj Ahmed. This is an open access article distributed under the Creative Commons Attribution License, which permits unrestricted use, distribution, and reproduction in any medium, provided the original work is properly cited. 
readers are referred to the survey paper [5] for acquaintance with the development of the theory of commuting maps and the various results that have been established.

In 2000, Beidar et al. [2] proved that a similar result holds true over $T_{r}(F)$, the ring of $r \times r$ upper triangular matrices over a field $F$. Their work showed that any linear commuting map $f: T_{r}(F) \rightarrow T_{r}(F)$ is again of the standard form, so $f(x)=\lambda x+\mu(x)$ for some $\lambda \in F$ and linear map $\mu: T_{r}(F) \rightarrow Z\left(T_{r}(F)\right)$. In [6] Cheung extended this result to triangular algebras.

Recently, in [3], Bounds extended some of these results to the case $N_{r}(F)$ - the ring of strictly upper triangular matrices over a field $F$ of characteristic zero. The author proved that if $f: N_{r}(F) \rightarrow N_{r}(F)$ is a commuting linear map, then there exists $\lambda \in F$ and an additive map $\mu: N_{r}(F) \rightarrow \Omega$ such that $f(x)=\lambda x+\mu(x)$ for all $x \in N_{r}(F)$, where $\Omega=\left\{a e_{1, r-1}+b e_{1, r}+c e_{2, r}: a, b, c \in F\right\}$ and $e_{i, j}$ denotes the standard matrix unit.

For a positive integer $m$, a map $f: R \rightarrow R$ is said to be m-power commuting if $\left[f(x), x^{m}\right]=0$ for all $x \in R$. Clearly, every commuting map is a 1-power commuting map. In [17] Brešar and Hvala studied 2-power commuting additive maps and showed that if $R$ is a prime ring with the extended centroid $C$, $\operatorname{char} R=2$ and $f: R \rightarrow R$ is a 2-power commuting additive map, then there exist $\lambda \in C$ and an additive map $\delta: R \rightarrow C$ such that $f(x)=\lambda x+\delta(x)$ for all $x \in R$. Later, Beidar et al. [18] extended this result to $m$-power commuting additive maps and proved that if $R$ is a prime ring with the extended centroid $C$, $\operatorname{char} R=0$ or $\operatorname{char} R>m$ and $f: R \rightarrow R$ is an m-power commuting additive map, then there exist $\lambda \in C$ and an additive map $\delta: R \rightarrow C$ such that $f(x)=\lambda x+\delta(x)$ for all $x \in R$. Recently, in [19], Liu and Yang characterize the $m$-power commuting additive maps on invertible or singular matrices.

In this paper, we examine $m$-power commuting additive maps over the ring of upper triangular matrices $T_{n}(R)$ over a commutative ring $R$ whose characteristic is not a divisor of $m$. Precisely, we will show that the following theorems are true.

Theorem 1. Let $R$ be a commutative ring. If $f: T_{n}(R) \rightarrow T_{n}(R)$ is an additive commuting map, then there exist $\lambda \in R$ and an additive map $\delta: T_{n}(R) \rightarrow R$ such that $f(X)=\lambda X+\delta(X) I_{n}$ for all $X \in T_{n}(R)$. 
Theorem 2. Let $R$ be a commutative ring whose characteristic is not a divisor of $m$. If $f: T_{n}(R) \rightarrow T_{n}(R)$ is an additive m-commuting map, then there exist $\lambda \in R$ and an additive map $\delta: T_{\infty}(R) \rightarrow R$ such that $f(X)=\lambda X+\delta(X) I_{n}$ for all $X \in T_{n}(R)$.

\section{Preliminaries and Commuting Additive Maps of $T_{n}(R)$}

We denote by $T_{n}(R)$ a set of upper triangular matrices over a commutive ring $R$. For $1 \leq i<j$ by $e_{i j}$ we mean the matrix unit - the matrix whose only nonzero entry is 1 in the $(i, j)$ th position. It is known that the product of $e_{i j}$ and $e_{k l}$ is equal to $e_{i j} \times e_{k l}=\delta_{j k} e_{i l}$, where $\delta_{i j}$ is the Kronecker delta.

For any $A=\left(a_{i j}\right) \in T_{n}(R)$, to abbreviate notation and facilitate calculations, we will write $A=\sum_{1 \leq i \leq j \leq n} a_{i j} e_{i, j}$. In particular, we put $I_{n}=\sum_{i=1}^{n} e_{i, i}$ and we write $Z\left(T_{n}(R)\right)$ for the center of $T_{n}(R), Z\left(T_{n}(R)\right)=R I_{n}$.

In order to prove our main result, we first need to establish following lemmas.

Lemma 2.1. Suppose that $f: T_{n}(R) \rightarrow T_{n}(R)$ is an additive map satisfying $X f(X)=f(X) X$ for all $\left.X \in T_{n}(R)\right)$. Then $f(X) Y+f(Y) X-X f(Y)-Y f(X)=0$ for all $X, Y \in T_{n}(R)$.

Lemma 2.2. Suppose that $f: T_{n}(R) \rightarrow T_{n}(R)$ is an additive map satisfying $X f(X)=f(X) X$ for all $X \in T_{n}(R)$ and $r \in R$. Then there exists $\lambda \in R$ such that $f\left(r I_{n}\right)=\lambda r I_{n}$.

Proof. Let $r \in R$ for every $X \in T_{n}(R), \quad f(X)\left(r I_{n}\right)+f\left(r I_{n}\right) X-X f\left(r I_{n}\right)-$ $\left(r I_{n}\right) f(X)=0$. Then $\left[f\left(r I_{n}\right), X\right]=0$. Thus, $f\left(r I_{n}\right) \in Z\left(T_{n}(R)\right)$. Consequently, there exists $\lambda \in R$ such that $f\left(r I_{n}\right)=\lambda r I_{n}$.

Lemma 2.3. Suppose that $f: T_{n}(R) \rightarrow T_{n}(R)$ is an additive map satisfying $X f(X)=f(X) X$ for all $X \in T_{n}(R)$. Then $f\left(\alpha e_{i i}\right)$ is a diagonal matrix with $\alpha \in R$, $f\left(\alpha e_{i i}\right) e_{i i}=e_{i i} f\left(\alpha e_{i i}\right)$ and $f\left(\alpha e_{i j}\right)\left(\alpha e_{i j}\right)=\left(\alpha e_{i j}\right) f\left(\alpha e_{i j}\right)$ for all distinct integers $i, j$ with $1 \leq i<j \leq n$. 
Proof. Write $f\left(\alpha e_{i i}\right)=\sum_{1 \leq r \leq s \leq n} a_{r s}^{i i}(\alpha) e_{r, s}$, where each $a_{r s}^{i i}: F \rightarrow F$ is an additive map for $1 \leq s \leq t \leq n$. We have $\left(\alpha e_{i i}\right) f\left(\alpha e_{i i}\right)=f\left(\alpha e_{i i}\right)\left(\alpha e_{i i}\right)$ and $\alpha \neq 0$. This implies that $f\left(\alpha e_{i i}\right) e_{i i}=e_{i i} f\left(\alpha e_{i i}\right)$.

Let $j$ be an integer such that $1 \leq j$ with $j \neq i$. Then we have $e_{i i} f\left(\alpha e_{i i}\right) e_{j j}=$ $e_{j j} f\left(\alpha e_{i i}\right) e_{i i}=0$. This implies $a_{i j}^{i i}(\alpha)=a_{i j}^{i i}(\alpha)=0$.

Let $j, k$ be an integer such that $1 \leq j, k$ with $j, k \neq i$ and $\alpha \neq 0$. For $X=\alpha e_{i i}$ and $Y=\beta e_{j j}$, we have

$$
f\left(\alpha e_{i i}\right)\left(\alpha e_{j j}\right)+f\left(\alpha e_{j j}\right)\left(\alpha e_{i i}\right)-\left(\alpha e_{i i}\right) f\left(\alpha e_{j j}\right)-\left(\alpha e_{j j}\right) f\left(\alpha e_{i i}\right)=0
$$

we obtain

$$
e_{i i} f\left(\alpha e_{j j}\right)+e_{j j} f\left(\alpha e_{i i}\right)=f\left(\alpha e_{j j}\right) e_{i i}+f\left(\alpha e_{i i}\right) e_{j j} .
$$

Multiplying by $e_{k k}$ from the left and by $e_{j j}$ from the right, we get $e_{k k} f\left(\alpha e_{i i}\right) e_{j j}=0$. This implies $a_{k j}^{i i}(\alpha)=0$. Thus $f\left(\alpha e_{i i}\right)$ is a diagonal matrix, as desired. Write $f\left(\alpha e_{i i}\right)=\sum_{1 \leq r \leq n} a_{r r}^{i i}(\alpha) e_{r, r}$. From $f\left(\alpha e_{i j}+I_{n}\right)\left(\alpha e_{i j}+I_{n}\right)=\left(\alpha e_{i j}+I_{n}\right) f\left(\alpha e_{i j}+I_{n}\right)$ we have $f\left(\alpha e_{i j}\right)\left(\alpha e_{i j}\right)=\left(\alpha e_{i j}\right) f\left(\alpha e_{i j}\right)$ and $\alpha \neq 0$. This implies $f\left(\alpha e_{i j}\right) e_{i j}=$ $e_{i j} f\left(\alpha e_{i j}\right)$.

Lemma 2.4. For all $\alpha \in R$, there exist $\lambda_{i} \in R, \delta_{i}: R \rightarrow R$ additive map such that $f\left(\alpha e_{i i}\right)=\lambda_{i} \alpha e_{i i}+\delta_{i}(\alpha) I_{n}$ for all integers $i$ with $i \geq 1$.

Proof. Write $f\left(\alpha e_{i i}\right)=\sum_{1 \leq r \leq n} a_{r r}^{i i}(\alpha) e_{r, r}$ for all distinct integers $i, j, k$ with $(k \leq l, \neq i)$, we have

$$
f\left(\alpha e_{i i}\right) \beta e_{k l}-\beta e_{k l} f\left(\alpha e_{i i}\right)+f\left(\beta e_{k l}\right) \alpha e_{i i}-\alpha e_{i i} f\left(\beta e_{k l}\right)=0 .
$$

Multiplying by $e_{k k}$ from the left and by $e_{l l}$ from the right, we get

$$
a_{k k}^{i i}(\alpha) \beta e_{k l}-\beta a_{l l}^{i i}(\alpha) e_{k l}=0 .
$$


This implies $a_{k k}^{i i}(\alpha)=a_{l l}^{i i}(\alpha)=\delta_{i}(\alpha)$, it follows that

$$
f\left(\alpha e_{i i}\right)=\sum_{1 \leq r \leq n} a_{r r}^{i i}(\alpha) e_{r, r}=\left(a_{i i}^{i i}(\alpha)-\delta_{i}(\alpha)\right) e_{i i}+\delta_{i}(\alpha) I_{n} .
$$

For all distinct integers $i, j$ with $(i<j)$ we have

$$
f\left(\alpha e_{i i}\right) \beta e_{i j}-\beta e_{i j} f\left(\alpha e_{i i}\right)+f\left(\beta e_{i j}\right) \alpha e_{i i}-\alpha e_{i i} f\left(\beta e_{i j}\right)=0 .
$$

Multiplying by $e_{i i}$ from the left and by $e_{j j}$ from the right, this implies

$$
a_{i i}^{i i}(\alpha) \beta e_{i j}-\beta \delta_{i}(\alpha) e_{i j}-\alpha e_{i i} f\left(\beta e_{i j}\right) e_{j j}=0,
$$

it follows that $a_{i i}^{i i}(\alpha) \beta-\beta \delta_{i}(\alpha)-\alpha a_{i j}^{i j}(\beta)=0$. Thus for $\beta=1$ we get

$$
a_{i i}^{i i}(\alpha)-\delta_{i}(\alpha)=\alpha a_{i j}^{i j}(1)=\alpha \lambda_{i j}=\alpha \lambda_{i}
$$

Hence $f\left(\alpha e_{i i}\right)=\alpha \lambda_{i} e_{i i}+\delta_{i}(\alpha) I_{n}$.

Lemma 2.5. For all $\alpha, \beta \in R$, for all distinct integers $i, j$ with $1 \leq i<j \leq n$, then there exists $\lambda \in R$, and the additives maps $\delta_{i}, a_{i i}^{i i}: R \rightarrow R$ such that $f\left(\alpha e_{i i}\right)=\lambda \alpha e_{i i}$ $+\delta_{i}(\alpha) I_{n}$ and $f\left(\beta e_{i j}\right)=\lambda \beta e_{i j}+a_{i i}^{i i}(\beta) I_{n}$.

Proof. Write $f\left(\alpha e_{i j}\right)=\sum_{1 \leq r \leq s \leq n} a_{r s}^{i i}(\alpha) e_{r, s}$, for all distinct integers $i, j$ with $1 \leq i<j \leq n$. Then by assumption,

$$
f\left(\alpha e_{i i}+\beta e_{i j}\right)\left(\alpha e_{i i}+\beta e_{i j}\right)=\left(\alpha e_{i i}+\beta e_{i j}\right) f\left(\alpha e_{i i}+\beta e_{i j}\right) .
$$

Hence,

$$
f\left(\beta e_{i j}\right) \alpha e_{i i}+f\left(\alpha e_{i i}\right) \beta e_{i j}=\alpha e_{i i} f\left(\beta e_{i j}\right)+\beta e_{i j} f\left(\alpha e_{i i}\right) .
$$

Multiplying by $e_{i i}$ from the left and by $e_{j j}$ from the right, this implies that

$$
e_{i i} f\left(\alpha e_{i i}\right) \beta e_{i j}=\alpha e_{i i} f\left(\beta e_{i j}\right) e_{j j}+\beta e_{i j} f\left(\alpha e_{i i}\right) e_{j j},
$$

we obtain $\lambda_{i} \beta=a_{i j}^{i j}(\beta)$. 
For $k<i$. Multiplying by $e_{k k}$ from the left and by $e_{i i}$ from the right, this implies $a_{i j}^{k i}(\beta)=0$.

For $i<l(l \neq j)$. Multiplying by $e_{i i}$ from the left and by $e_{l l}$ from the right, this implies $a_{i j}^{i l}(\beta)=0$.

Similarly, using $f\left(\alpha e_{j j}+\beta e_{i j}\right)\left(\alpha e_{j j}+\beta e_{i j}\right)=\left(\alpha e_{j j}+\beta e_{i j}\right) f\left(\alpha e_{j j}+\beta e_{i j}\right)$ we have $\beta \lambda_{j}=a_{i j}^{i j}(\beta)$. Hence $\beta \lambda_{j}=a_{i j}^{i j}(\beta)=\beta \lambda_{i}$.

We notice $\lambda=\lambda_{i}=\lambda_{j}$. In particular, $f\left(\alpha e_{i i}\right)=\lambda \alpha e_{i i}=\delta_{i}(\alpha) I_{\infty}$ for $k<j$, $(k \neq i), \quad$ by assumption, $f\left(\beta e_{i j}\right) \alpha e_{j j}+f\left(\alpha e_{j j}\right) \beta e_{i j}=\alpha e_{j j} f\left(\beta e_{i j}\right)+\beta e_{i j} f\left(\alpha e_{j j}\right)$. Multiplying by $e_{k k}$ from the left and by $e_{j j}$ from the right, hence $f\left(\beta e_{i j}\right) \alpha e_{i i}$ $+f\left(\alpha e_{i i}\right) \beta e_{i j}=\alpha e_{i i} f\left(\beta e_{i j}\right)+\beta e_{i j} f\left(\alpha e_{i i}\right)$.

Multiplying by $e_{i i}$ from the left and by $e_{j j}$ from the right, this implies that $a_{i j}^{k j}(\beta)=0$.

Similarly, using for $j<l,(l \neq i)$ multiplying by $e_{j j}$ from the left and by $e_{l l}$ from the right, it follows that $a_{i j}^{j l}(\beta)=0$.

Let $l<k$ and $\notin\{i, j\}$. From $f\left(e_{k k}+\alpha e_{i j}\right)\left(e_{k k}+\alpha e_{i j}\right)=\left(e_{k k}+\alpha e_{i j}\right) f\left(e_{k k}+\alpha e_{i j}\right)$ and multiplying by $e_{l l}$ from the left and by $e_{k k}$ from the right, we get $a_{i j}^{l k}(\beta)=0$.

Hence $f\left(\beta e_{i j}\right)=a_{i j}^{i j}(\beta) e_{i j}+\sum_{1 \leq r} a_{r r}^{i i}(\beta) e_{r, r}$.

From $f\left(\beta e_{i j}\right)\left(\alpha e_{i j}\right)=\left(\beta e_{i j}\right) f\left(\alpha e_{i j}\right)$, multiplying by $e_{i i}$ from the left and by $e_{j j}$ from the right, we get $a_{i i}^{i j}(\beta)=a_{j j}^{i j}(\beta)$.

From $f\left(\alpha e_{i j}+\beta e_{l j}\right)\left(\alpha e_{i j}+\beta e_{l j}\right)=\left(\alpha e_{i j}+\beta e_{l j}\right) f\left(\alpha e_{i j}+\beta e_{l j}\right)$. Multiplying by $e_{l l}$ from the left and by $e_{j j}$ from the right, we get $a_{l l}^{i j}(\alpha) \beta=\beta a_{j j}^{i j}(\alpha)$. Then $f\left(\beta e_{i j}\right)=a_{i j}^{i j}(\beta) e_{i j}+a_{i i}^{i j}(\beta) I_{n}=f\left(\beta e_{i j}\right)=\beta \lambda e_{i j}+a_{i i}^{i j}(\beta) I_{n}$. 
Proof of Theorem 1. Suppose that $f: T_{n}(R) \rightarrow T_{n}(R)$ is an additive map satisfying $X f(X)=f(X) X$ for all $X \in T_{n}(R)$. We put $X=\sum_{1 \leq i \leq j \leq n} a_{i j} e_{i, j}$, then

$$
\begin{aligned}
f(X) & =f\left(\sum_{1 \leq i \leq j \leq n} x_{i j} e_{i, j}\right) \\
& =\sum_{1 \leq i \leq j \leq n} f\left(x_{i j} e_{i, j}\right) \\
& =\sum_{1 \leq i \leq n} f\left(x_{i i} e_{i, i}\right)+\sum_{1 \leq i<j \leq n} f\left(x_{i j} e_{i, j}\right) \\
& =\sum_{1 \leq i \leq n}\left(\lambda x_{i i} e_{i i}+\delta_{i}\left(x_{i i}\right) I_{n}\right)+\sum_{1 \leq i<j \leq n} x_{i j} \lambda e_{i j}+a_{i i}^{i j}\left(x_{i j}\right) I_{n} \\
& =\lambda X+\sum_{1 \leq i \leq n} \delta_{i}\left(x_{i i}\right) I_{n}+\sum_{1 \leq i<j \leq n} a_{i j}^{i j}\left(x_{i j}\right) I_{n} \\
& =\lambda X+\delta(X) I_{n} .
\end{aligned}
$$

This proves Theorem 1 .

\section{3. $m$-commuting Additive Maps of $T_{n}(R)$}

Lemma 3.1. Let $m$ be a natural number and let $R$ be a commutative ring whose characteristic is not a divisor of $m$. Suppose that $f: T_{n}(R) \rightarrow T_{n}(R)$ is an additive map satisfying $X^{m} f(X)=f(X) X^{m}$ for all $X \in T_{n}(R)$ and $r \in R$, then $f\left(r I_{n}\right) \in$ $Z\left(T_{n}(R)\right)$.

Proof. Let $\alpha, \beta \in R$. Moreover, let $i<j$. Clearly,

$$
\left(\alpha e_{i j}+\beta I_{\infty}\right)^{m} f\left(\alpha e_{i j}+\beta I_{n}\right)=\left(\alpha e_{i j}+\beta I_{\infty}\right)^{m} f\left(\alpha e_{i j}+\beta I_{n}\right)
$$

This implies

$$
\left(m \alpha \beta^{m-1} e_{i j}+\beta^{m} I_{n}\right) f\left(\alpha e_{i j}+\beta I_{n}\right)=f\left(\alpha e_{i j}+\beta I_{n}\right)\left(m \alpha \beta^{m-1} e_{i j}+\beta^{m} I_{n}\right) .
$$


Hence

$$
e_{i j} f\left(\alpha e_{i j}+\beta I_{n}\right)=f\left(\alpha e_{i j}+\beta I_{n}\right) e_{i j}
$$

Similarly, using $r, \alpha, \beta \in R$ we obtain

$$
e_{i j} f\left(\alpha e_{i j}+(\beta+r) I_{n}\right)=f\left(\alpha e_{i j}+(\beta+r) I_{n}\right) e_{i j} .
$$

The difference of above two relations yields $e_{i j} f\left(r I_{n}\right)=f\left(r I_{n}\right) e_{i j}$, then $f\left(r I_{n}\right) \in$ $Z\left(T_{n}(R)\right)$.

Lemma 3.2. Let $m$ be a natural number and let $R$ be a commutative ring whose characteristic is not a divisor of $m$. Suppose that $f: T_{n}(R) \rightarrow T_{n}(R)$ is an additive map satisfying $X^{m} f(X)=f(X) X^{m}$ for all $X \in T_{n}(R)$, then $X f(X)=f(X) X$ for all $X \in T_{n}(R)$.

Proof. We have $X^{m} f(X)=f(X) X^{m}$ for every $X \in T_{n}(R)$. Clearly,

$$
\left[f(X),\left(X+p I_{n}\right)^{m}\right]+\left[f\left(p I_{n}\right),\left(X+p I_{n}\right)^{m}\right]=0 .
$$

Recall that $f\left(r I_{n}\right) \in Z\left(T_{n}(R)\right)$. Thus,

$$
\sum_{1 \leq k \leq m \leq n} p^{m-k} C_{m}^{k}\left[f(X), X^{k}\right]=0 .
$$

Using matrix notation we can rewrite these systems in the following way: For $p=1, p=2, \ldots, p=m-1$

$$
\left(\begin{array}{ccccc}
1 C_{m}^{1} & 1 C_{m}^{2} & 1 C_{m}^{3} & \cdots & 1 C_{m}^{m} \\
2^{m-1} C_{m}^{1} & 2^{m-2} C_{m}^{2} & 2^{m-3} C_{m}^{3} & \cdots & 1 C_{m}^{m} \\
3^{m-1} C_{m}^{1} & 3^{m-2} C_{m}^{2} & 3^{m-3} C_{m}^{3} & \cdots & 1 C_{m}^{m} \\
\vdots & \vdots & \vdots & \vdots & \vdots \\
m^{m-1} C_{m}^{1} & m^{m-2} C_{m}^{2} & m^{m-3} C_{m}^{3} & \cdots & 1 C_{m}^{m}
\end{array}\right)\left(\begin{array}{c}
{[f(X), X]} \\
{\left[f(X), X^{2}\right]} \\
{\left[f(X), X^{3}\right]} \\
\vdots \\
{\left[f(X), X^{m}\right]}
\end{array}\right)=0 .
$$

Because the determinant of the Vandermonde matrix formed by the coefficients of the system is not zero, we get that $[f(X), X]=0$. This proves Theorem 2 . 


\section{References}

[1] H. E. Bell and W. S. Martindale, Centralizing mappings of semiprime rings, Canad. Math. Bull. 30 (1987), 92-101. https://doi.org/10.4153/CMB-1987-014-x

[2] K. I. Beidar, M. Brešar and M. A. Chebotar, Functional identities on upper triangular matrix algebras, J. Math. Sci. 102 (2000), 4557-4565. https://doi.org/10.1007/BF02673884

[3] J. Bounds, Commuting maps over the ring of strictly upper triangular matrices, Linear Algebra Appl. 507 (2016), 132-136. https://doi.org/10.1016/j.laa.2016.05.041

[4] M. Brešar, Centralizing mappings and derivations in prime rings, J. Algebra 156 (1993), 385-394. https://doi.org/10.1006/jabr.1993.1080

[5] M. Brešar, Commuting maps: a survey, Taiwanese J. Math. 8 (2004), 361-397. https://doi.org/10.11650/twjm/1500407660

[6] W.-S. Cheung, Commuting maps of triangular algebras, J. London Math. Soc. 63 (2001), 117-127. https://doi.org/10.1112/S0024610700001642

[7] W. Franca, Commuting maps on some subsets of matrices that are not closed under addition, Linear Algebra Appl. 437 (2012), 388-391.

https://doi.org/10.1016/j.laa.2012.02.018

[8] W. Franca, Commuting maps on rank- $k$ matrices, Linear Algebra Appl. 438 (2013), 28132815. https://doi.org/10.1016/j.laa.2012.11.013

[9] W. Franca and N. Louza, Commuting maps on rank-1 matrices over noncommutative division rings, Comm. Algebra 45 (2017), 4696-4706.

https://doi.org/10.1080/00927872.2016.1278010

[10] M. Hongan and A. Trzepizur, On generalization of a theorem of Posner, Math. J. Okayama Univ. 27 (1985), 19-23.

[11] J. Mayne, Centralizing mappings of prime rings, Canad. Math. Bull. 27 (1984), 122-126. https://doi.org/10.4153/CMB-1984-018-2

[12] C. R. Miers, Centralizing mappings of operator algebras, J. Algebra 59 (1979), 56-64. https://doi.org/10.1016/0021-8693(79)90152-2

[13] E. C. Posner, Derivations in prime rings, Proc. Amer. Math. Soc. 8 (1957), 1093-1100. https://doi.org/10.1090/S0002-9939-1957-0095863-0

[14] R. Słowik, Expressing infinite matrices as products of involutions, Linear Algebra Appl. 438 (2013), 399-404. https://doi.org/10.1016/j.laa.2012.07.032 
[15] J. Vukman, Commuting and centralizing mappings in prime rings, Proc. Amer. Math. Soc. 109 (1990), 47-52. https://doi.org/10.1090/S0002-9939-1990-1007517-3

[16] X. Xu, Commuting maps on rank-k matrices, Electron. J. Linear Algebra 27 (2014), 735741. https://doi.org/10.13001/1081-3810.1958

[17] M. Brešar and B. Hvala, On additive maps of prime rings, Bull. Austral. Math. Soc. 51 (1995), 377-381. https://doi.org/10.1017/S0004972700014209

[18] K. L. Beidar, Y. Fong, P.-H. Lee and T.-L. Wong, On additive maps of prime rings satisfying the engel condition, Comm. Algebra 25 (1997), 3889-3902.

https://doi.org/10.1080/00927879708826093

[19] C.-K. Liu and J.-J. Yang, Power commuting additive maps on invertible or singular matrices, Linear Algebra Appl. 530 (2017), 127-149.

https://doi.org/10.1016/j.laa.2017.04.038 